\title{
Análisis multitemporal de la laguna Suches y del vigor de la vegetación del bofedal de Huaytire, Tacna
} \author{
Tacna \\ ${ }^{1, a}$ José Francisco Chambe Bahamondes \\ ${ }^{1, b}$ Dariella Sharyley Quintana Calizaya \\ ${ }^{1, c}$ Marianela Sanga Franco
}

Multi-temporary analysis of the lagoon Suches and vegetation vigor of peatland Huaytire,

\section{ARTÍCULO ORIGINAL \\ ${ }^{1}$ Universidad Nacional Jorge Basadre Grohmann, Facultad de Ciencias Agropecuarias, Semillero de investigación de Tecnología Ambiental. Tacna, Perú.}

\section{Correspondencia:}

dquintanac@unjbg.edu.pe

aRCID: 0000-0002-4623-2419 bRCID: 0000-0001-7293-0117 'ORCID: 0000-0002-4118-7321

Palabras clave: Cambio climático, extracción de agua, imágenes satelitales, NDVI, NDWI, pastoreo

Keywords: Climate change, water extraction, satellite images, NDVI, NDWI, livestock grazing

Información adicional

Presentado: 02/05/2021

Aprobado: 17/05/2021

\section{RESUMEN}

La laguna Suches y el bofedal de Huaytire, ubicados en la provincia de Candarave del departamento de Tacna, son ecosistemas importantes que brindan diversos servicios ecosistémicos. En esta investigación, se determinó el cambio del área del espejo de agua de la laguna Suches y el cambio de los valores de NDVI del bofedal de Huaytire entre los años 1975 a 2020, mediante el análisis de imágenes satelitales obtenidas del Servicio Geológico de los Estados Unidos - USGS (Landsat 2, Landsat 5, Landsat 7 y Landsat 8). Se calculó el Índice Normalizado Diferencial de Vegetación (NDVI) y el Índice Diferencial de Agua Normalizado (NDWI). Los resultados evidencian una disminución notable del área de la laguna Suches y del vigor de la vegetación, este último indicando una disminución de la cobertura del bofedal de Huaytire. Aunque, no fue posible establecer una relación causal con los factores que estarían ocasionando tal disminución, la evidencia científica revisada sugiere que el cambio climático, la derivación y extracción de agua superficial y subterránea; así como, el pastoreo de ganado camélido, serían las causantes de los resultados encontrados.

\section{ABSTRACT}

The lagoon Suches and the peatland Huaytire, located in the province of Candarave in the department of Tacna, are important ecosystems that provide various ecosystem services. In this research, the change in the area of the water mirror of the lagoon Suches and the change in the NDVI values of the peatland Huaytire between 1975 and 2020 were determined by analyzing satellite images obtained from the United States Geological Survey - USGS (Landsat 2, Landsat 5, Landsat 7 and Landsat 8). The Normalized Difference Vegetation Index (NDVI) and the Normalized Difference Water Index (NDWI) were calculated. The results show a notable decrease in the area of the Suches lagoon and in the vegetation vigor, the latter indicating a decrease in the cover of the peatland Huaytire. Although it was not possible to establish a causal relationship with the factors causing this decrease, the scientific evidence reviewed suggests that climate change, surface and groundwater diversion and extraction, as well as camelid livestock grazing, would be responsible for the results found. 


\section{INTRODUCCIÓN}

Son muchos los problemas ambientales ocasionados por las actividades antrópicas, siendo uno de ellos el deterioro y pérdida de los ecosistemas (Salamanca Gómez, 2018). Este problema ambiental no solo representa una amenaza para la biodiversidad (Van Dover et al., 2017); sino también, para las poblaciones locales que se benefician de los servicios ecosistémicos (Sonter et al., 2018), particularmente poblaciones que utilizan los recursos de manera directa. Sumado a ello, el cambio climático es otra amenaza que acentúa aún más la pérdida y deterioro de los ecosistemas (Paula et al., 2018), debido a cambios en la precipitación y temperatura principalmente (Jiang et al., 2017).

El Perú es un país caracterizado por una alta diversidad biológica, no solo a nivel de especies; sino también, a nivel de ecosistemas y a nivel genético (Ames et al., 2019). Sin embargo, mucha de esta diversidad se ha ido perdiendo a lo largo de los años, a pesar de los diversos esfuerzos que a nivel gubernamental se han hecho (May et al., 2012). En ese sentido, ecosistemas como los bosques de la Amazonía (Rojas et al., 2019), los lagos (Rudolph et al., 2006), los bofedales (Sulca et al., 2019), las lomas costeras (Sotomayor \& Jiménez, 2008), los bosques altoandinos (Zutta et al., 2012), los pastizales (Yaranga et al., 2018), entre otros, se encuentran actualmente amenazados. Particularmente, en los ecosistemas de las zonas altoandinas, el pastoreo junto con la minería son las principales causas de deterioro (Domic et al., 2018; Polk et al., 2019; Rolando et al., 2017). Sumado a ello, el cambio climático es un factor que podría actuar simultáneamente con estas actividades y acentuar el efecto en zonas de mayor altitud (Meneses et al., 2014).

Uno de los ecosistemas de mucha importancia en las zonas altoandinas son los bofedales, caracterizados por tener una vegetación perenne y cuya principal fuente de agua es de origen subterráneo, el mismo que se origina de las lluvias y deshielo de los glaciares (Garcia \& Otto, 2015). Los bofedales son importantes sumideros de carbono (Anthelme et al., 2018), hábitat de una gran diversidad de especies silvestres (Galindo \& Anaya, 2018) y mitigan procesos de erosión (Bonnesoeur et al., 2019). A pesar de los diversos servicios ecosistémicos que ofrecen, históricamente han sufrido diversas amenazas antrópicas como el sobrepastoreo (Domic et al., 2018), derivación del agua para la agricultura (Rolando et al., 2017), extracción de aguas subterráneas (Elmore et al., 2006). Además, son ecosistemas vulnerables a la variabilidad de la temperatura y precipitación (Imfeld et al., 2021; Leng et al., 2019); no obstante, son limitados los estudios orientados al monitoreo temporal de estos ecosistemas, los mismos que ayudarían a determinar la tasa de cambio y la identificación de posibles agentes de cambio.

Por otra parte, los lagos y lagunas altoandinas también son ecosistemas de importancia por concentrar especies ícticas endémicas (Vila et al., 2007), por la regulación del microclima (Rolando et al., 2017) y por ser una importante fuente de abastecimiento de agua para la población local (Adrian et al., 2009). Sin embargo, son sistemas que igualmente soportan una fuerte presión antrópica y son vulnerables frente al cambio climático (Michelutti et al., 2015). Por ejemplo, la evacuación de aguas residuales inadecuadamente tratadas ha conllevado a procesos de eutrofización severos (Duwig et al., 2014) y el aumento de la temperatura por el cambio climático ha conducido a una reestructuración de las comunidades biológicas (Michelutti et al., 2015). En la zona altiplánica las proyecciones bajo diferentes escenarios de cambio climático indican un aumento de la temperatura y disminución de las precipitaciones (Rolando et al., 2017). Esto se traduciría en una disminución del flujo de agua que ingresa a estos cuerpos de agua y un aumento de la evaporación, disminuyendo la profundidad de estos ecosistemas.

El uso de la teledetección y los sistemas de información geográfica (SIG) como herramienta digital permiten estudiar los sistemas ecológicos a diversas escalas espaciales y temporales para dar una mejor información, proyección del uso y manejo de los ecosistemas (Flórez et al., 2017) siendo útiles para cartografiar y monitorear los recursos (Wu, 2017). El análisis de imágenes satelitales mediante herramientas de teledetección permite, de manera relativamente sencilla y económica, el monitoreo espacial y temporal de los ecosistemas (e.g. Baniya et al., 2018; Guirado et al., 2019). Particularmente, el desarrollo de algunos índices como el índice Normalizado de la Vegetación (NDVI por sus siglas en inglés) y el Índice Diferencial 
de Agua Normalizado (NDWI por sus siglas en inglés), permiten el análisis de la vegetación y cuerpos de agua respectivamente. El NDVI, al utilizar la longitud de onda del rojo e infrarrojo cercano, tiene la habilidad de diferenciar entre la cobertura vegetal y otro tipo de cobertura. Por otra parte, el NDWI, al utilizar la banda verde e infrarrojo cercano, permite la diferenciación de cuerpos de agua. Los valores de estos índices están en función de la energía absorbida o reflejada por las superficies, ya sea vegetación o cuerpos de agua, en diversas partes del espectro electromagnético (Verhulst \& Govaerts, 2010). Entonces, ambos índices, pueden ser utilizados para el monitoreo de ecosistemas para la detección temprana de anomalías y posterior identificación de los posibles agentes causantes. De hecho, son más frecuentes los estudios orientados al monitoreo de diversos ecosistemas con la aplicación de herramientas de teledetección (e.g. Guirado et al., 2019; Pang et al., 2017)

En la región de Tacna, el bofedal de Huaytire y la laguna Suches representan una fuente importante de forraje y de recursos hídricos. Sin embargo, son ecosistemas que estarían fuertemente impactados por actividades antrópicas como la extracción de agua subterránea, pastoreo extensivo (Franco, 2013) y agricultura (ANA, 2010). Esto podría afectar el sistema hidrológico y el equilibrio de ambos ecosistemas. Además, los bofedales (Dangles et al., 2017) y las lagunas (Adrian et al., 2009), son considerados centinelas efectivos del cambio climático por su sensibilidad al clima y respuesta rápida al cambio; de modo que, su evaluación debiera ser prioritario. No obstante, hasta el momento no se han hecho estudios cuantitativos orientados a la evaluación temporal de estos ecosistemas. Por ello, los objetivos fueron: (a) evaluar el cambio del área del espejo de agua de la laguna Suches y (b) evaluar el cambio de los valores de NDVI del bofedal de Huaytire durante los años 1975 a 2020.

\section{MATERIALES Y MÉTODOS}

\section{Área de estudio}

El análisis se llevó acabo en la región de Tacna, provincia de Candarave en el bofedal de Huaytire y laguna Suches. El área de estudio está a $4600 \mathrm{~m} \mathrm{s.} \mathrm{n.} \mathrm{m.,} \mathrm{en} \mathrm{las} \mathrm{coordenadas} 16^{\circ} 55^{\prime} 41.84^{\prime \prime}$ de latitud sur y $70^{\circ}$ $23^{\prime} 41.10^{\prime \prime}$ de longitud oeste. La estación lluviosa es entre los meses de enero a marzo, con precipitaciones que van desde $10-100 \mathrm{~mm}$ y con temperaturas que oscilan entre los 1.2 a $17^{\circ} \mathrm{C}$ (Franco, 2013). El clima de la zona es frío con variaciones de temperatura entre el día y la noche, con fuertes vientos helados y continuas nevadas en época de lluvias (Sulca et al., 2019).

El bofedal de Huaytire, caracterizado por comunidades vegetales dominados por Distichia muscoides y Oxychloe andina (Franco \& Sulca, 1996), es aprovechado por las comunidades campesinas aledañas ya que constituyen la base de la crianza de camélidos como las alpacas y llamas. Los camélidos sudamericanos en la región Tacna son criados por las comunidades de las zonas altoandinas, una de las principales fuentes de ingreso económico (Gobierno Regional de Tacna, 2016).

La laguna Suches se encuentra en la provincia de Candarave entre dos flancos del volcánico Barroso. Esta laguna se alimenta de los ríos Huaytire y Livecalane y por las filtraciones de los bofedales de la pampa de Huaytire. La laguna Suches es un área importante de nidificación de especies como Podiceps occipitalis, Chloephaga melanoptera y Fulica gigantea(Moreno, 1996). 


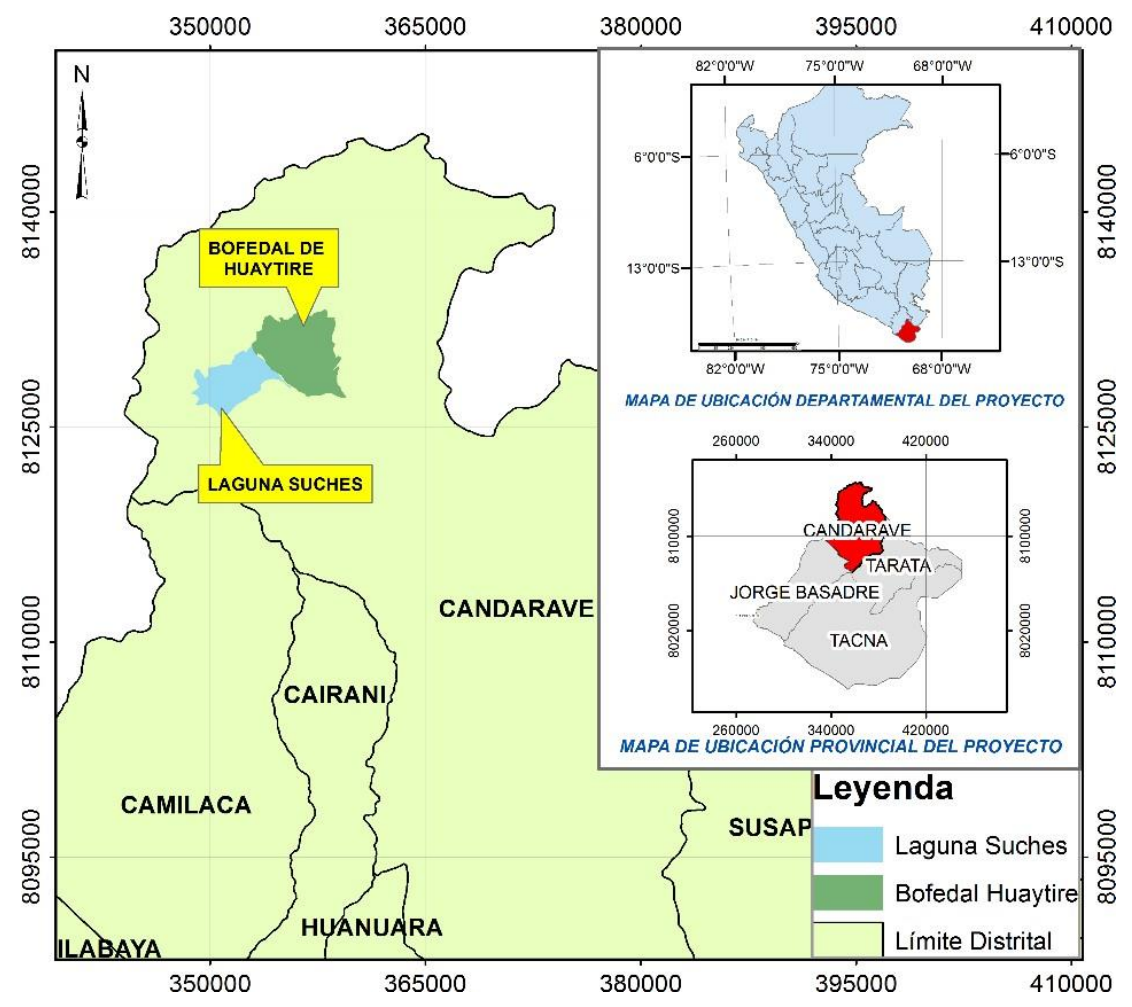

Figura 1. Área de estudio de los bofedales de Huaytire y la laguna Suches de Tacna.

\section{Colección de datos}

La información satelital fue obtenida del Servicio Geológico de los Estados Unidos - USGS de los satélites Landsat 2, Landsat 5, Landsat 7 y Landsat 8. Estos satélites están equipados con sensores ópticos que permiten tener una visión amplia a distintas longitudes de onda del globo terráqueo. De acuerdo al área de estudio identificada, se eligió el Path 07 y Row 72 para la descarga de datos para los años 1975, 1986, 1990, 1994,1996,1999,2005,2015 y 2020 del servidor de la USGS EarthExplorer (https://earthexplorer.usgs.gov/). Para la selección de las imágenes satelitales se priorizó aquellas que tenían un porcentaje de nubosidad $<5 \%$ y que se encuentren entre los meses de junio y agosto, meses que corresponden a la estación seca (Tabla 1).

\section{Tabla 1}

Información satelital obtenida de la Laguna Suches y los bofedales de Huaytire de Tacna entre los años 1975 a 2020.

\begin{tabular}{ccccccc}
\hline Nombre de Información Satelital & Año & Mes & Día & Satélite & Path & Row \\
\hline LM02_L1TP_002072_19750625_20180425_01_T2 & 1975 & Junio & 25 & Landsat 02 & 2 & 72 \\
\hline LT05_L1TP_002072_19860705_20170217_01_T1 & 1986 & Julio & 05 & Landsat 05 & 2 & 72 \\
\hline LT05_L1TP_002072_19900622_20170131_01_T1 & 1990 & Junio & 22 & Landsat 05 & 2 & 72 \\
\hline LC05_L1TP_002072_19960801_20170103_01_T1 & 1996 & Agosto & 01 & Landsat 05 & 2 & 72 \\
\hline LE07_L1TP_003072_19990725_20170217_01_T1 & 1999 & Julio & 25 & Landsat 07 & 2 & 72 \\
\hline LT05_L1TP_002072_20050719_20161128_01_T1 & 2005 & Julio & 19 & Landsat 05 & 2 & 72 \\
\hline LT05_L1TP_002072_20100723_20161014_01_T1 & 2010 & Julio & 23 & Landsat 05 & 2 & 72 \\
\hline LC08_L1TP_002072_20150721_20170406_01_T1 & 2015 & Julio & 21 & Landsat 08 & 2 & 72 \\
\hline LC08_L1TP_002072_20200718_20200722_01_T1 & 2020 & Julio & 18 & Landsat 08 & 2 & 72 \\
\hline
\end{tabular}




\section{Preprocesamiento y validación}

El preprocesamiento de las imágenes satelitales se realizó a través del software Qgis v. 3.12.2, con el plugin Semi-Automatic Classification Plugin v. 5.3.6.1, que permite obtener una imagen satelital adecuada para la aplicación de índices espectrales. Además, permite la corrección atmosférica por el método DOS1 que consiste en corregir el efecto de dispersión de energía electromagnética en las partículas de agua suspendidas en la atmósfera, permitiendo de esta manera, restar de la imagen los valores que estas agregan a los datos de la imagen. Finalmente, se realizó el recorte de cada una de las imágenes satelitales obtenidas de acuerdo al área de estudio delimitada.

\section{Cálculo de Índices}

\section{NDVI}

El índice de vegetación más conocido y usado es el Índice Normalizado Diferencial de Vegetación (NDVI Normalized Difference Vegetation Index). El cálculo del NDVI - (Pang et al., 2017b) implica el uso una fórmula con dos bandas, el Infrarrojo Cercano (NIR) y el rojo (RED) la cual se muestra continuación:

$$
\mathrm{NDVI}=\frac{\mathrm{NIR}-\mathrm{Red}}{\mathrm{NIR}+\mathrm{Red}}
$$

\section{NDWI}

El índice NDWI (Índice Diferencial de Agua Normalizado) podemos identificar masas de agua y zonas con elevada humedad. El cálculo del NDWI implica el uso una fórmula con dos bandas, el verde (GREEN) y el Infrarrojo Cercano (NIR) la cual se muestra continuación (Farías \& Lagos, 2015):

$$
\text { NDVI }=\frac{\text { Green }- \text { NIR }}{\text { Green }+ \text { NIR }}
$$

El cálculo del NDVI y NDWI fue realizado a través de la herramienta CALCULADORA RÁSTER en el software Qgis. Los valores del NDVI fluctúan entre -1 (coberturas sin vegetación) y 1 (vegetación densa y sana). En los bofedales valores de NDVI por encima de 0.25 indican presencia de vegetación, y cuanto más alto sea el valor de este índice, las condiciones de vigor son mejores (Alvarado et al., 2019). Por otro lado, los valores de NDWI superiores a 0 indican la presencia de un cuerpo de agua.

\section{Análisis de datos}

Se aplicaron pruebas de regresión entre los años de evaluación y el NDVI y NDWI en el programa R v. 4.0.4.

\section{RESULTADOS}

El análisis multitemporal, durante los años de 1975 hasta 2020, permitió observar la reducción del área del espejo de agua y cambio en su forma de la laguna de Suches (Figura 2). Se encontró una relación negativa entre el área de la laguna Suches y los años de evaluación $\left(R^{2}=0.39, p=0.04\right)$ (Figura 3). En 1975 el área del espejo de agua en la laguna Suches fue 1508.39 ha, mientras que en el 2020 fue 1150.12 ha. En el 2015 se registró una muy importante reducción del área de la laguna (822.082ha) en comparación a los demás años en evaluación (Figura 3). 

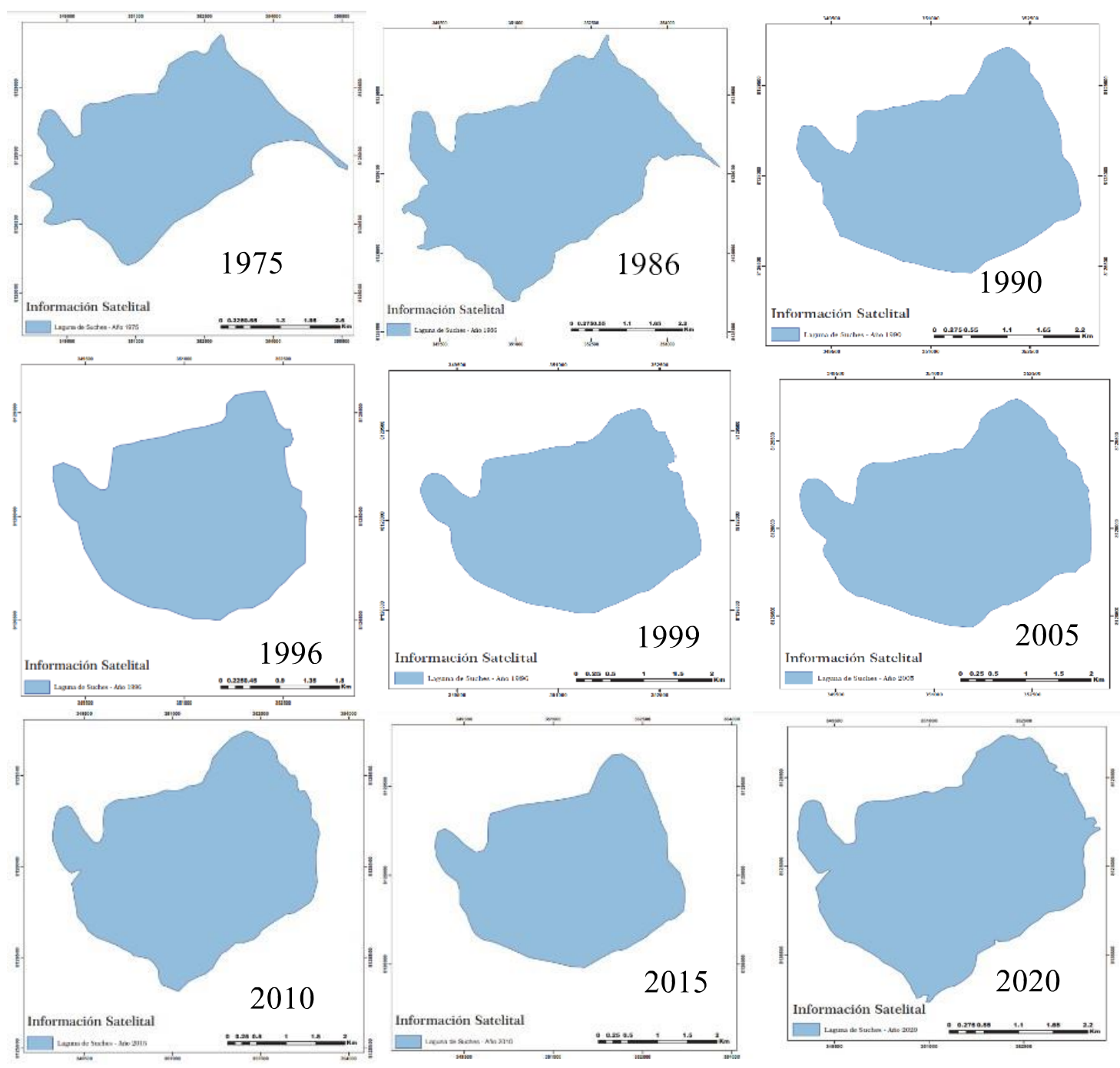

Figura 2. Superficie de la laguna Suches (ha) entre los años 1975 a 2020.
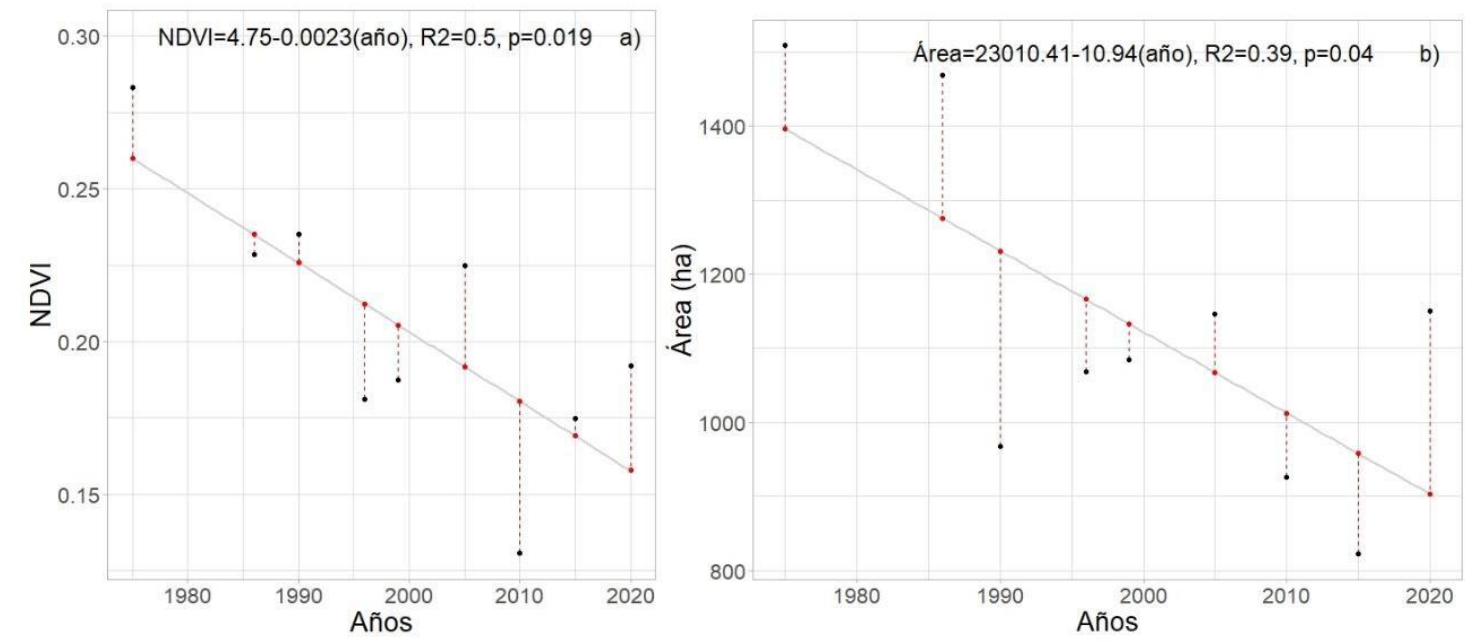

Figura 3. Variación temporal del NDVI del bofedal de Huaytire (a) y área de la laguna Suches (b) entre los años 1975 a 2020, Tacna. 
Se encontró una relación negativa entre los valores del NDVI y los años de valuación $\left(\mathrm{R}^{2}=0.5, \mathrm{p}=0.019\right)$, indicando una disminución en el vigor de la vegetación (Figura 3). En general, se observa una alta variación de los valores de NDVI que van desde una media de 0.28 (1975) a 0.192 (2020), registrándose el más bajo valor en el 2010 (0.131) (Figura 5). En el 2020, valores de NDVI altos (alrededor de 0.5) se restringe a un pequeño parche del bofedal de Huaytire, en tanto que en 1975 gran parte del bofedal evidenciaba altos niveles de vigor de la vegetación (Figura 4).

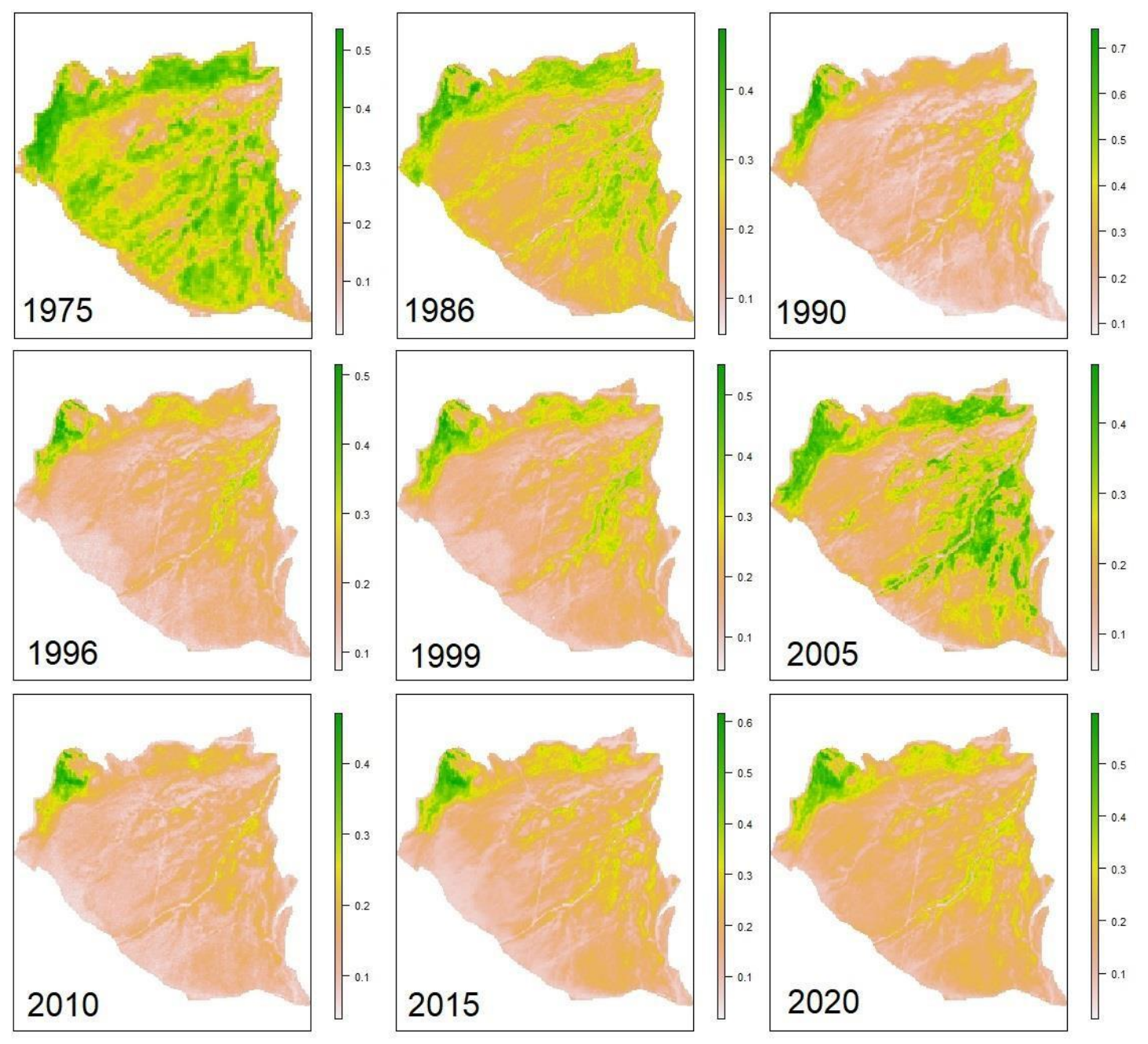

Figura 4. Superficie de los bofedales de Huaytire entre los años de 1975 a 2020 durante los meses junio a agosto. 


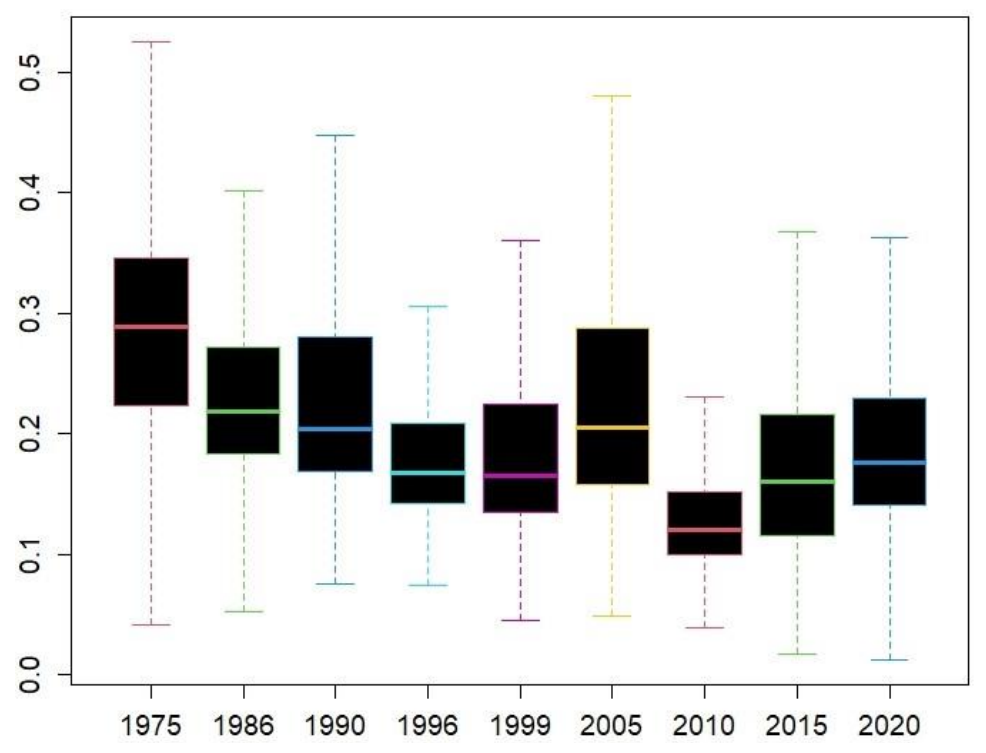

Figura 5. Diagrama de cajas mostrando los valores de NDVI entre los años 1975 a 2020 en el bofedal de Huaytire, Tacna.

\section{DISCUSIÓN}

Los resultados obtenidos producto del análisis multitemporal de la laguna Suches y de los bofedales de Huaytire han mostrado una reducción del área y disminución del vigor de la vegetación respectivamente. Son muchos los factores que podrían estar influyendo en estos cambios, los mismos que son motivos de discusión.

La tendencia de disminución de las lagunas es un fenómeno que se puede evidenciar en distintas partes del mundo (e.g. Castañeda, 2014, Yap, 2015), asociado sobre todo al cambio climático. Las proyecciones de cambio climático en la zona altiplánica del Perú indican un aumento de la temperatura y una disminución de las precipitaciones (Imfeld et al., 2021; Rolando et al., 2017), conduciendo al aumento de la temperatura en el epilimnio de cuerpos de agua como las lagunas (Elo et al., 1998; Winslow et al., 2014) y mayores niveles de evaporación (Wongchuig et al., 2018); de modo que, la profundidad de las lagunas altoandinas sería cada vez menor. El cambio de la temperatura ocasiona la alteración del nivel del agua subterránea y, en consecuencia, implica un cambio en la descomposición de la vegetación aledaña y de la capacidad de retención del agua (Leng et al., 2019). Además, el aumento de la temperatura implica la reestructuración de las comunidades biológicas (Michelutti et al., 2015) y un aumento de la productividad biológica en el epilimnio (Elo etal., 1998).

Por otra parte, además de la presión que estaría ejerciendo el cambio climático, actividades antrópicas también podrían estar influyendo en la disminución del nivel de agua de la laguna Suches. En las zonas altoandinas del Perú un problema importante tiene que ver con el limitado acceso al agua; y es en estas zonas donde la actividad minera está ampliamente desarrollada. Por ello, históricamente diversos conflictos sociales han suscitado entre la actividad minera y las poblaciones locales por el acceso al agua (Salem et al., 2018). La empresa Southern Corporation que opera la mina Toquepala en la región de Tacna, desde inicios de su funcionamiento ha contado con licencias de uso de agua superficial y subterránea para extraer el recurso hídrico de las cabeceras de cuencas de esa región (Pérez-Jiménez, 2018). Estas zonas de extracción se encuentran tanto en la laguna Suches (Autoridad Nacional del Agua, 2010), como en el bofedal de Huaytire. Esta actividad podría implicar un incremento del efecto sobre los ecosistemas que el cambio climático (Stefanova et al., 2019). Sin embargo, son necesarios mayores estudios que permitan determinar relaciones causales con el cambio climático y extracción de agua; de modo que, se pueda dilucidar el 
porcentaje de disminución del área de laguna atribuible al cambio climático y a la extracción del agua.

En este estudio se evidenció una disminución de los valores de NDVI; esto es una disminución del vigor de la vegetación del bofedal de Huaytire, siendo contrario a lo encontrado en otros estudios (e.g. Baniya et al., 2018; Pang et al., 2017, Pauca - Tanco et al., 2020). Se ha evidenciado que los cambios del NDVI serían más afectados por los cambios de la temperatura que por la precipitación (Pang et al., 2017a). Entonces, en condiciones de mayores temperaturas, que es lo pronosticado bajo un escenario de cambio climático (Rolando et al., 2017), la vegetación crecería en mejores condiciones ambientales, debiendo traducirse en una mayor productividad y biomasa (Baniya et al., 2018). Sin embargo, en el bofedal de Huaytire se observa una muy importante reducción del NDVI desde 1975 al 2020, evidenciando la degradación y pérdida de este ecosistema. Esto puede estar relacionado a actividades como el pastoreo; así como, a la extracción del agua para actividades mineras.

En el bofedal se pasta hasta 12000 animales entre alpacas y llamas que provocan el sobrepastoreo (Franco \& Sulca, 2019). El sobrepastoreo, además de la disminución de la cobertura vegetal, implica un cambio en la composición y estructura de las comunidades vegetales (Polk et al., 2019), promoviendo el establecimiento de especies exóticas (Domic et al., 2018). Por otra parte, la extracción de agua implica una reducción del nivel de la napa freática (Elmore et al., 2006; Guirado et al., 2019), ocasionando la reducción de la cobertura vegetal (Huang et al., 2019), particularmente de las especies con raíces superficiales (Elmore et al., 2006). Sin embargo, este efecto se ve afectado por la textura de los suelos; de modo que, texturas finas de suelo implican una mayor retención de agua proveniente de las precipitaciones (Gokdemir et al., 2019); y, por lo tanto, un menor efecto de la disminución de la nata freática. Por otra parte, la disminución de la napa freática desencadena un proceso de oxidación de la biomasa que típicamente se acumula en los bofedales, conllevando al incremento de la emisión de gases de efecto invernadero (Humpenöder et al., 2020).

\section{CONCLUSIONES}

Los resultados obtenidos en la laguna Suches y los bofedales de Huaytire no son atípicos en contraste a los acontecimientos mundiales, encontrándose dentro de lo esperado. Se determinó el cambio del área del espejo de agua de la laguna Suches $(23.75 \%$ ) y el cambio de los valores de NDVI del bofedal de Huaytire (31.43\%), evidenciándose una importante reducción entre los años 1975 a 2020. Aunque no es posible establecer una relación causal con el cambio climático, pastoreo y extracción de agua en la zona de estudio, es indudable que estos estarían ocasionando algún efecto sobre los ecosistemas evaluados. Entonces, es necesario el desarrollo de mayores estudios que permitan establecer la tasa de cambio; así como, los factores que incidirían en estos cambios. Además, el monitoreo temporal de estos ecosistemas altoandinos debiera ser prioritario, puesto que ambos ecosistemas actúan como centinelas de los efectos del cambio climático (Adrian et al., 2009; Dangles et al., 2017).

\section{AGRADECIMIENTOS}

Este trabajo se desarrolló como parte de las actividades de investigación del Semillero de Investigación de Tecnología Ambiental de la Facultad de Ciencias Agropecuarias de la Universidad Nacional Jorge Basadre Grohmann. Los autores expresan su agradecimiento a la docente Ivon Gutierrez Flores, tutora del semillero de investigación, por los conocimientos impartidos y el apoyo incondicional para el desarrollo del presente estudio. 


\section{REFERENCIAS}

Adrian, R., O'Reilly, C. M., Zagarese, H., Baines, S. B., Hessen, D. O., Keller, W., Livingstone, D. M., Sommaruga, R., Straile, D., Van Donk, E., Weyhenmeyer, G. A., \& Winder, M. (2009). Lakes as sentinels of climate change. Limnology and Oceanography, 54(6 PART 2), 2283-2297. https://doi.org/10.4319/lo.2009.54.6_part_2.2283

Alvarado, C., Leandro, A., Mayor, N., \& Marcos, D. S. (2019). Comparison of Vegetation Indexes with Landsat images using cloud computing : Pampa de Majes-Siguas area, Arequipa-Peru. Revista de Investigación de Física, 22(1), 27-34.

Ames, F., Quispe, H., Zuñiga, D., Segovia, M., \& Kessler, M. (2019). Bosques de Polylepis: Biodiversidad en la Región Central del Perú. https://repositorio.continental.edu.pe/handle/20.500.12394/5922

Anthelme, F., Meneses, I., \& Dangles, O. (2018). Métodos para estudiar el efecto del cambio climático sobre los bofedales y sus servicios ambientales inherentes. Ecología en Bolivia, 49(3). http://bibliotecadigital.ciren.cl/handle/123456789/26589

Autoridad Nacional del Agua. (2010). Estudio evaluación de recursos hídricos cuencas de los rios Locumba y Sama: Vol. I.

Baniya, B., Tang, Q., Huang, Z., Sun, S., \& Techato, K. anan. (2018). Spatial and temporal variation of NDVI in response to climate change and the implication for carbon dynamics in Nepal. Forests, 9 , 1-18. https://doi.org/10.3390/f9060329

Bonnesoeur, V., Locatelli, B., Guariguata, M. R., Ochoa-Tocachi, B. F., Vanacker, V., Mao, Z., Stokes, A., \& Mathez-Stiefel, S. L. (2019). Impacts of forests and forestation on hydrological services in the Andes: A systematic review. Forest Ecology and Management, 433(December 2018), 569-584. https://doi.org/10.1016/j.foreco.2018.11.033

Castañeda, E. (2014). Análisis Multitemporal Del Cuerpo De Agua De La Laguna El Sonso Mediante Imágenes Satelitales Landsat. Universidad Militar Nueva Granda (Bogotá), 20.

Dangles, O., Rabatel, A., Kraemer, M., Zeballos, G., Soruco, A., Jacobsen, D., \& Anthelme, F. (2017). Ecosystem sentinels for climate change? Evidence of wetland cover changes over the last 30 years in the tropical Andes. PLoS ONE, 12(5), 1-22. https://doi.org/10.1371/journal.pone.0175814

Domic, A., Capriles, J., Escobar-Torrez, K., Santoro, C., \& Maldonado, A. (2018). Two thousand years of land-use and vegetation evolution in the Andean Highlands of Northern Chile inferred from pollen and charcoal analyses. Quaternary, 1(3), 32. https://doi.org/10.3390/quat1030032

Duwig, C., Archundia, D., Lehembre, F., Spadini, L., Morel, M. C., Uzu, G., Chincheros, J., Cortez, R., \& Martins, J. M. F. (2014). Impacts of anthropogenic activities on the contamination of a sub watershed of Lake Titicaca. Are antibiotics a concern in the Bolivian Altiplano? Procedia Earth and Planetary Science, 10, 370-375. https://doi.org/10.1016/j.proeps.2014.08.062

Elmore, A. J., Manning, S. J., Mustard, J. F., \& Craine, J. M. (2006). Decline in alkali meadow vegetation cover in California: The effects of groundwater extraction and drought. Journal of Applied Ecology, 43(4), 770-779. https://doi.org/10.1111/j.1365-2664.2006.01197.x

Elo, A.-R., Huttula, T., Peltonen, A., \& Virta, J. (1998). The effects of climate change on the temperature conditions of lakes. Boreal Environment Research, 3, 137-150.

Farías, M., \& Lagos, M. (2015). Estimación de vegetación, humedad superficial y cuerpos de agua utilizando percepción remota: Salar Brinkerhoff, desierto de Atacama, Chile. ResearchGate, october. https://doi.org/10.13140/RG.2.1.1390.1526

Flórez, G., Rincon, A., \& Santiago, P. (2017). Análisis multitemporal de las coberturas vegetales en el área de influencia de las minas de oro ubicadas en la parte alta del sector de Maltería en Manizales, Colombia.DYNA, 84(201), 95-101 https://doi.org/10.15446/dyna.v84n201.55759

Franco León, P., \& Sulca Quispe, L. (2019). Evaluación Socio - Ambiental del Bofedal Huaytire de la Provincia de Candarave - Tacna. Ciencia \& Desarrollo, 12, 93-98. https://doi.org/10.33326/26176033.2008.12.259 
Galindo, A., \& Anaya, M. (2018). Hidroquímica y su variabilidad espacio temporal en un bofedal altoandino de la Reserva Paisajística Nor Yauyos Cochas, Perú. Idesia, 36, (4). https://doi.org/10.4067/s071834292018005002603

Garcia, E., \& Otto, M. (2015). Caracterización ecohidrológica de humedales alto andinos usando imágenes de satélite multitemporales en la cabecera de cuenca del río Santa, Ancash, Perú. Ecología Aplicada, 14(1-2), 115. https://doi.org/10.21704/rea.v14i1-2.88

Gobierno Regional de Tacna. (2016). Memoria Descriptiva del mapa de uso actual de tierras de la Región de Tacna.

Gokdemir, C., Rubin, Y., Li, X., Li, Y., \& Xu, H. (2019). Vulnerability analysis method of vegetation due to groundwater table drawdown induced by tunnel drainage. Advances in Water Resources, 133(March), 103406. https://doi.org/10.1016/j.advwatres.2019.103406

Guirado, E., Blanco-Sacristán, J., Rigol-Sánchez, J. P., Alcaraz-Segura, D., \& Cabello, J. (2019). A multitemporal object-based image analysis to detect long-lived shrub cover changes in drylands. Remote Sensing, 11(22), 1-17. https://doi.org/10.3390/rs11222649

Huang, F., Zhang, D., \& Chen, X. (2019). Vegetation response to groundwater variation in arid environments: Visualization of research evolution, synthesis of response types, and estimation of groundwater threshold. International Journal of Environmental Research and Public Health, 16(10). https://doi.org/10.3390/ijerph16101849

Humpenöder, F., Karstens, K., Lotze-Campen, H., Leifeld, J., Menichetti, L., Barthelmes, A., \& Popp, A. (2020). Peatland protection and restoration are key for climate change mitigation. Environmental Research Letters, 15(10). https://doi.org/10.1088/1748-9326/abae2a

Imfeld, N., Sedlmeier, K., Gubler, S., Correa Marrou, K., Davila, C. P., Huerta, A., Lavado-Casimiro, W., Rohrer, M., Scherrer, S. C., \& Schwierz, C. (2021). A combined view on precipitation and temperature climatology and trends in the southern Andes of Peru. International Journal of Climatology, 41(1), 679-698. https://doi.org/10.1002/joc.6645

Jiang, R., Gan, T. Y., Xie, J., Wang, N., \& Kuo, C. C. (2017). Historical and potential changes of precipitation and temperature of Alberta subjected to climate change impact: 1900-2100. Theoretical and Applied Climatology, 127(3-4), 725-739. https://doi.org/10.1007/s00704-015-1664-y

Leng, L. Y., Ahmed, O. H., \& Jalloh, M. B. (2019). Brief review on climate change and tropical peatlands. Geoscience Frontiers, 10(2), 373-380. https://doi.org/10.1016/j.gsf.2017.12.018

May, R. Von, Catenazzi, A., Angulo, A., \& Venegas, P. J. (2012). Investigación y conservación de la biodiversidad en Perú: importancia del uso de técnicas modernas y procedimientos administrativos eficientes. Revista Peruana de Biología, 19(3), 351-358.

Meneses, R., Loza Herrera, S., Lliully, A., Palabral, A., \& Anthelme, F. (2014). Métodos para cuantificar diversidad y productividad vegetal de los bofedales frente al cambio climático. Ecología en Bolivia, 49(3), 42-55.

Michelutti, N., Wolfe, A. P., Cooke, C. A., Hobbs, W. O., Vuille, M., \& Smol, J. P. (2015). Climate change forces new ecological states in tropical Andean lakes. PLoS ONE, 10(2), 1-10. https://doi.org/10.1371/journal.pone.0115338

Moreno, T. (1996). Lagunas Altoandinas Del Sur Del Perú: Características Químicas. Ciencia \& Desarrollo, 3, 89-95.

Pang, G., Wang, X., \& Yang, M. (2017a). Using the NDVI to identify variations in, and responses of, vegetation to climate change on the Tibetan Plateau from 1982 to 2012. Quaternary International, 444,1-10. https://doi.org/10.1016/j.quaint.2016.08.038

Pang, G., Wang, X., \& Yang, M. (2017b). Using the NDVI to identify variations in, and responses of, vegetation to climate change on the Tibetan Plateau from 1982 to 2012. Quaternary International, 444, 87-96. https://doi.org/10.1016/j.quaint.2016.08.038

Paula, P. A., Zambrano, L., \& Paula, P. (2018). Multitemporal Analysis of vegetation change at Chimborazo $\mathrm{R}$ e serveas aresultof c li matechange. Enfoque UTE, 2,125-137. http://ingenieria.ute.edu.ec/enfoqueute/ 
Pérez-Jiménez, S. (2018). Impacto ambiental de la Compañía minera Southern Perú Cooper Corporation en América Latina: Una aproximación histórica de comienzos del siglo XX a la actualidad. Revista Geográfica de América Central, 3(61E), 489-503. https://doi.org/10.15359/rgac.61-3.25

Polk, M. H., Young, K. R., Cano, A., \& León, B. (2019). Vegetation of andean wetlands (bofedales) in $\mathrm{h}$ u a s c a rá n a t i o n a $1 \mathrm{p}$ a r k, P e r u. MiresandPe $a t, 24,1-26$. https://doi.org/10.19189/MaP.2018.SNPG.387

Rojas, N., Barboza, E., Maicelo, L., Oliva, S., \& Salas, R. (2019). Deforestación en la Amazonía peruana: índices de cambios de cobertura y uso del suelo basado en SIG. Boletín de la Asociación de Geógrafos Españoles, 2538(81), 1-34.https://doi.org/10.21138/bage.2538a

Rolando, J. L., Turin, C., Ramírez, D. A., Mares, V., Monerris, J., \& Quiroz, R. (2017). Key ecosystem services and ecological intensification of agriculture in the tropical high-Andean Puna as affected by land-use and climate changes. Agriculture, Ecosystems and Environment, 236, 221-233. https://doi.org/10.1016/j.agee.2016.12.010

Rudolph, D. L., Sultan, R., Garfias, J., \& McLaren, R. G. (2006). Significance of enhanced infiltration due to groundwater extraction on the disappearance of a headwater lagoon system: Toluca Basin, Mexico. Hydrogeology Journal, 14(1-2), 115-130. https://doi.org/10.1007/s10040-005-0463-4

Salamanca Gómez, M. Á. (2018). Análisis multitemporal sobre la pérdida del espejo de agua sobre el humedal laguna La Herrera por efectos antrópicos asociados a la minería.

Salem, J., Amonkar, Y., Maennling, N., Lall, U., Bonnafous, L., \& Thakkar, K. (2018). An analysis of Peru: Is w a terd riving mining conflict s? Resources Polic y, $58,1-9$. https://doi.org/10.1016/j.resourpol.2018.09.010

Sonter, L. J., Ali, S. H., \& Watson, J. E. M. (2018). Mining and biodiversity: Key issues and research needs in conservation science. Proceedings of the Royal Society B: Biological Sciences, 285(1892). https://doi.org/10.1098/rspb.2018.1926

Sotomayor Melo, D. A., \& Jiménez Milón, P. (2008). Condiciones Meteorológicas y Dinámica Vegetal del Ecosistema Costero Lomas de Atiquipa (Caravelí - Arequipa) en el Sur eel Perú. Ecología Aplicada, 7(1-2), 1.https://doi.org/10.21704/rea.v7i1-2.353

Stefanova, A., Hesse, C., Krysanova, V., \& Volk, M. (2019). Assessment of Socio-Economic and Climate Change Impacts on Water Resources in Four European Lagoon Catchments. Environmental Management, 64(6), 701-720. https://doi.org/10.1007/s00267-019-01188-1

Sulca, L., Franco, P., \& Oyague, E. (2019). Caracterización trófica de dos bodefales de la provincia de Candarave, región Tacna. Ciencia \& Desarrollo, 16(21), 37-49. https://doi.org/10.33326/26176033.2017.21.728

Van Dover, C. L., Ardron, J. A., Escobar, E., Gianni, M., Gjerde, K. M., Jaeckel, A., Jones, D. O. B., Levin, L. A., Niner, H. J., Pendleton, L., Smith, C. R., Thiele, T., Turner, P. J., Watling, L., \& Weaver, P. P. E. (2017). Biodiversity loss from deep-sea mining. Nature Geoscience, 10(7), 464-465. https://doi.org/10.1038/ngeo2983

Verhulst, N., \& Govaerts, B. (2010). The normalized difference vegetation index (NDVI) GreenSeeker TM handheld sensor: Toward the integrated evaluation of crop management Part A: Concepts and case $s t u d i$ e s. 16. https://repository.cimmyt.org/bitstream/handle/10883/550/94192.pdf?sequence=1\&isAllowed=y

Vila, I., Pardo, R., \& Scott, S. (2007). Freshwater fishes of the Altiplano. Aquatic Ecosystem Health and Management, 10(2), 201-211.https://doi.org/10.1080/14634980701351395

Winslow, L. A., Read, J. S., Hansen, G. J. A., \& Hanson, P. C. (2014). Small lakes show muted climate change signal in deepwater temperatures. Geophysical Research Letters, 42, 355-361. https://doi.org/10.1002/2014GL062325.Received

Wongchuig, S. C., Mello, C. R., \& Chou, S. C. (2018). Projections of the impacts of climate change on the water deficit and on the precipitation erosive indexes in Mantaro River Basin, Peru. Journal of Mountain Science, 15(2), 264-279. https://doi.org/10.1007/s11629-017-4418-8 
Wu, Q. (2017). GIS and Remote Sensing Applications in Wetland Mapping and Monitoring. Comprehensive Geographic Information Systems, 3(September 2017), 140-157. https://doi.org/10.1016/B978-012-409548-9.10460-9

Yap,A. (2015). Análisis multitemporal de glaciares y lagunas. Pontifica Universidad Católica Del Peru.

Yaranga, R., Custodio, M., Chanamé, F., \& Pantoja, R. (2018). Floristic diversity in grasslands according to plant formation in the Shullcas river sub-basin, Junin, Peru. Scientia Agropecuaria, 9(4), 511-517. https://doi.org/10.17268/sci.agropecu.2018.04.06

Zutta, B. R., Rundel, P. W., Saatchi, S., Casana, J. D., Gauthier, P., Soto, A., Velazco, Y., \& Buermann, W. (2012). Prediciendo la distribución de Polylepis: bosques Andinos vulnerables y cada vez más importantes. Revista Peruana de Biología, 19(2), 205-212. https://doi.org/10.15381/rpb.v19i2.849 